\title{
SUBJETIVIDAD Y DONACIÓN EN JEAN-LUC MARION
}

\section{Roberto J. Walton*}

Jean-Luc Marion ha propuesto una reducción a la donación para avanzar más allá de la reducción epistémica que se limita a la objetividad y la reducción ontológica que concierne al ser de los entes. Se trata de una donación que tan solo a través de una respuesta adecuada puede revelar la excedencia dada. Marion pretende dar cuenta de fenómenos saturados en que lo dado intuitivamente supera los anticipable por medio de significados y lo manifestable como objeto o ente. Un caso privilegiado es el fenómeno erótico que se da con la reducción erótica del ego al amante. Esta reducción pasa por tres estadios en los cuales pregunto si se me ama, me decido a amar como el primero que ama, y descubro finalmente que he sido amado desde siempre. La reducción lleva al tratamiento de fenómenos en que "cada uno da al otro lo que no posee por sí mismo, pero de lo cual sin embargo dispone solo para el otro; así cada uno, en su propia penuria de sí mismo respecto de sí mismo, se revela sin embargo más íntimo para el otro que este otro para sí mismo" 1 . Así, una donación que recibo del otro y por la que el otro me interpela converge con la realización de la propia subjetividad.

\section{Reducción epistémica y reducción erótica}

Según Marion, la reducción epistémica es la reducción que los objetos, y el ego como un objeto más, son referidos al sujeto. Mientras que la certeza de los objetos puede desvanecerse, la autocerteza del ego es inconmovible. No obstante, encierra dos problemas. Por un lado, la certeza se refiere a objetos, y el ego no tiene el modo de ser de los objetos sino el modo de ser del poder ser y la apertura de mundo. Por el otro, la certeza es un producto del ego, que se autocertifica del mismo modo que certifica los objetos. Por estar referida a un objeto y resultar de una autocertificación, la certeza está afectada de vanidad.

\footnotetext{
"Investigador CONICET. Profesor de la Universidad Católica de Santa Fe y de la Universidad de Buenos Aires. grwalton@fibertel.com.ar

${ }^{1}$ Marion, Jean-Luc, Le phénoméne erotique, Grasset, Paris, 2003, p. 327.
} 
Solo puede darme seguridad contra la vanidad aquello que me sobrepasa radicalmente, esto es, el ser amado. De ahí que, como primera formulación de la reducción erótica, la pregunta "¿se me ama de otra-parte?" deba reemplazar a la pregunta "¿soy yo?". Por tanto, a la certeza de símismo a partir de sí-mismo proporcionada por la reducción epistémica se contrapone una seguridad venida de otra-parte. Esta seguridad da lugar a una transformación de la ipseidad en virtud de la cual me descubro afectado. Si la pregunta "¿se me ama de otra-parte?" tiene una respuesta positiva, el advenimiento del otro me conmueve del modo más íntimo, y lo mismo sucede si tiene una respuesta negativa. Me encuentro ahí donde me afecta un posible en-otra-parte.

Marion considera imposible responder a la pregunta " $\mathrm{ise}$ me ama de otra-parte?" por medio del amor a sí mismo y la ilusión de perseverar en el propio ser. Por eso es necesario radicalizar la reducción erótica mediante una segunda formulación: “ipuedo amar, yo el primero?". Un primer momento de esta reducción erótica radicalizada consiste en la decisión de hacer como si yo amara el primero en un avance que procede por anticipación sin suponer reciprocidad. Con la decisión de amar que toma el amante que avanza antes de que el otro aparezca, se da una intuición que tiene el carácter de una tonalidad afectiva amorosa y se incrementa en la medida en que el amante progresa hasta convertirse en una intuición saturante. Esta intuición está polarizado hacia un otro virtual, y es ciega porque carece de significado.

\section{El significado y el juramento}

La definición habitual de fenómeno como lo que plenifica un significado a través de la intuición no puede aplicarse al otro. En primer lugar, en la reducción erótica radicalizada, decido amar por avance, y esta decisión provoca desde mí solamente una tonalidad afectiva como mi experiencia intuitiva. Mis vivencias amorosas solo confirman mi condición de amante, pero no hacen visible al otro que amo. El problema no consiste en confirmar por intuición un significado vacío sino en fijar un significado preciso a la intuición sobreabundante y vaga que provoca la decisión de amar. En segundo lugar, el significado debe fijar mi intuición y de ese modo hacerme manifiesto el otro como un fenómeno de pleno derecho. En lugar de representarme el otro y rebajarlo a un objeto que podría constituir, el 
significado debe hacerme experienciar una radical alteridad. $\mathrm{Ha}$ de ofrecerme lo que la intuición vaga que produzco por mi decisión no proporciona. Puesto que no experiencio esta alteridad en mi avance, debo hacerlo en el advenimiento sobre mí de un significado provocado no por mi intencionalidad sino por una contra-intencionalidad.

En la reducción erótica, por tanto, el fenómeno del otro se distingue de los demás porque la intuición y el correspondiente significado se relacionan de un modo diferente con el ego. Mientras que la intuición de amar me pertenece, el significado del otro me adviene desde un en-otraparte. Este significado es el rostro del otro, que no tiene un contenido intuitivo especial, $y$, sin embargo, se apodera de mí mediante la contraintencionalidad de su mirada. No puedo conferirle ni los significados que atribuyo a objetos y entes, ni los vagos significados que despliega mi intuición de amante. Por el contrario, el rostro del otro me significa de un modo constrictivo "No matarás". Pero no se limita a exigirme esto de un modo universal y negativo, sino que responde a mi rostro de un modo positivo y en persona por medio de un “jHeme aquí!". Marion insiste en que el fenómeno amoroso, que no se constituye a partir del ego, no es un fenómeno común ni se compone de dos fenómenos distintos, sino que es un "fenómeno cruzado" o "fenómeno de doble entrada" que tiene el privilegio de hacer visible la comunión de una dualidad de dos intuiciones irreductibles fijadas por un único significado.

A la decisión de hacer "como si" yo amara el primero, se sigue como un segundo momento la recepción del significado del otro en el común "¡Heme aquí!" del juramento. La promesa es la misma de modo que dos juramentos se superponen en virtud del significado puramente formal de la exposición que se pone a disposición del otro en un abandono del ego en nominativo. Cada uno se deja interpelar y convocar en vocativo por el otro, y este aparece como un atributario en dativo de una asignación y exposición.

\section{La carne}

Al juramento sigue como tercer momento la erotización de cada carne por la otra. Mi cuerpo difiere de las cosas físicas inertes porque tiene la condición de carne por la que puede sentir las cosas y ante todo sentirse. $\mathrm{Su}$ heteroafección solo es posible porque a la vez se siente en una autoafección. Además de las cosas inertes y mi carne, existe otra carne cuyo 
sentir no puedo sentir yo en mi propia carne porque en tal caso la relegaría a cosa física. $Y$ aunque pudiera sentirla sin convertirla en cuerpo, ella desaparecería como otra carne porque se identificaría con la mía. Tras descartar tanto la reducción de la otra carne a cosa como la identificación de los sentires, Marion procura dilucidar el modo en que puedo sentir la otra carne en mi carne. Experiencio la otra carne como tal porque, a diferencia de las cosas físicas, no me resiste sino que me hace un lugar en ella dejándose invadir. En una doble pasividad, la recepción de mi carne por la carne del otro me da mi propia carne erotizada, a la vez que doy su propia carne erotizada al otro por medio de mi carne. En este cruce, el otro me da lo que no tiene, es decir, mi carne, y yo le doy lo que no tengo, es decir, su carne. Esto significa que la erotización ofrece a la carne de ambos su única fenomenalización posible. Al darme inmediatamente mi carne erotizada, la carne del otro se manifiesta en mi propia carne. Pero no aparece directamente como cosa $u$ objeto sino indirectamente, según la fenomenalidad de su carne, como aquello que me fenomenologiza a mí mismo en tanto carne erotizada.

Marion se refiere a un "experienciar (se) experienciar (se)" como el modo de fenomenalización que es propio del cruce de carnes. Se lo puede desplegar en los siguientes componentes: (i) siento mi carne en una autoafección como condición de posibilidad de todo sentir ulterior, (ii) siento la carne del otro ya no en una autoafección sino en tanto me hace un lugar en ella, (iii) experiencio que la carne del otro me siente en tanto le hago un lugar en mí, (iv) experiencio que la carne del otro experiencia que yo la siento, (v) experiencio que, en tanto siento la carne del otro, yo siento que ella me siente; y (vi) experiencio que, en tanto la carne del otro me siente, ella siente que yo la siento. (i) y (ii) corresponden a lo que yo siento acerca de mí y del otro, (iii) y (iv) a lo que el otro siente acerca de sí y de mí, y (v) y (vi) al entrelazamiento de los respectivos sentires. Se añaden dos precisiones. En primer lugar, el cruce de carnes depende del juramento sin el cual ni el otro ni yo podríamos asumirlo racionalmente, pero, por otro lado, da consistencia al juramento: "[...] sin esta erotización compartida, el juramento común permanecería como un comportamiento lingüístico abstracto, que no se fenomenologiza en ninguna parte, y no me individualizaría como tampoco a ningún otro"2 . En segundo lugar, la erotización no es una

2 Ibid., p. 192. 
cosa ni un espectáculo visible. Por eso solo ofrece indicios de ella misma, y se puede simular que se tiene o no la experiencia de ella.

Según Marion, el amor se caracteriza por su univocidad de modo que no es posible diferenciar éros y agápe, y este sentido unívoco se extiende a múltiples figuras. Como figura particular, la sexualidad se impone con agudeza, pero no es la más significativa. Así, al señalar que el rostro testimonia la realización sin resto de la carne como una "carne en gloria" o "carne glorificada", Marion da dos ejemplos: "Miremos la carne de una joven parturienta que tiene al niño recién nacido en sus brazos, carne que remonta hasta su rostro en que se encarnan indistintamente el sufrimiento que desaparece, el placer difuso y la alegría que se vuelve sobre sí. Miremos al Resucitado (y no siempre tan solo el cadáver del Crucificado) en el retablo de Isenheim, donde el rostro emblanquece y casi desaparece, salvo los ojos, en la gloria que sumerge su carne -desde ahora definitivamente viva, irresistible por haber sabido no resistir, aun a la peor muerte. El rostro erotizado también recapitula toda su carne; en su mirada, veo -si es que veo algo- la ola irreprimible de su carne que surge en él, que lo da a sí mismo por primera vez"3.

\section{El gozo}

En el rostro erotizado veo la carne individualizada del otro en tanto ella se siente y se experiencia en una trascendencia que la convierte en inaccesible para mí. Su mirada muestra la experiencia de su carne que yo le doy, y que puedo darle precisamente porque no la experiencio. A su vez, él ve que me convierto en mi carne. Se establece una comunión en la que cada uno aparece en el otro sin confundirse con él. Así, las carnes se cruzan sin confusión ni mezcla en un único fenómeno amoroso. Las carnes son irreductibles porque cada una surge de su sentir. Cada una da a la otra lo que no tiene. Tampoco hay separación porque las dos carnes experimentan la misma realización erótica. Hay una inmanencia y trascendencia recíproca por la cual cada uno ve en el otro el mismo experienciar(se) experienciar (se). Marion contrapone el gozo del otro al uso del otro. No gozo de mi placer sino del suyo, y, si gozo también, este placer solo es un reflejo del suyo. Si no gozara en el gozo del otro, simplemente no gozaría.

${ }^{3}$ Ibid., p. 199 s. 
El fenómeno erótico cesa, y esta suspensión de la erotización implica dejar de proporcionarle una carne al otro que ya no me da su propia carne. Al desaparecer las carnes reaparecen los cuerpos físicos como entes del mundo. Pero la carne del otro y la mía no desaparecen de la misma manera. En mi caso, al cesar la erotización, no pierdo mi carne sino el acceso en ella a otra carne. El fin de la erotización priva a mi carne de la otra carne, pero no le quita su autoexperienciarse, de modo que subsiste una carne de amante sin erotización en acto. Por tanto, hay tres niveles que corresponden al cuerpo, a la carne y a la carne erotizada. En el caso del otro, la condición intermedia de una carne ni corporizada ni erotizada escapa a la fenomenalización porque solo accedo a la carne del otro en su erotización por mi carne y en mi erotización por su carne. Solo así puedo sentir que el otro tiene rango de carne, es decir, que él me siente como yo lo siento en el modo del experienciar(se) experienciar(se).

Según Marion, al cesar la erotización se pierde todo criterio fenomenológico para ver en otro cuerpo físico una carne. Solo quedan argumentos probables basados en la analogía, la empatía, el lenguaje, etc., que no manifiestan efectivamente el fenómenos del otro, y solo permiten presumirlo. Sin la erotización, y sin experienciar (se) (en) lo que no le resiste, mi carne queda limitada a lo que le resiste, es decir, a los objetos del mundo, $y$, por tanto, también se desvanece. Del mismo modo que no hay verdadero recuerdo del sueño, no hay memoria del gozo porque no hay objetos estables que puedan ser reconstituidos. La relación visible de los cuerpos físicos es ajena al invisible cruce de las carnes.

\section{La persona naturalizada}

Solo la carne decide acerca del desencadenamiento y fin de la erotización. La finitud del amante se manifiesta en que su carne se erotiza por sí misma y lo convierte en un autómata sujeto a una pasividad radical. Así como el ego cogito teme que una facultad desconocida piense en él, el amante teme que la carne se erotice sin el juramento. Además de esta primera finitud relativa implicada en la autonomía de la carne automática, hay una finitud intrínseca que tiene que ver con los límites de la erotización ya que la carne no puede erotizarse al infinito. En tal caso, la erotización nos arrancaría definitivamente del mundo. Por el contrario, la suspensión de la erotización nos retiene en el mundo y hace posible una ulterior historia 
erótica. El amante quiere lo infinito, pero la erotización de las carnes cruzadas es finita. Esta separación entre el amante y su erotización se manifiesta en primer lugar como finitud temporal en la suspensión de la erotización.

La separación temporal se consolida como una separación espacial entre mi carne que se convierte en cuerpo para el otro y su carne que se convierte en cuerpo para mí. Aquí surge la sospecha porque cabe preguntarse si efectivamente ha tenido lugar la erotización de la carne. Puesto que mi erotización no me enseña nada acerca del gozo del otro, sospecho que no hubo otra carne sino otro cuerpo. Con esto se desvanece tanto la fenomenalización del otro como el gozo de mi propia individualidad que yo recibo en la erotización. La sospecha se extiende al punto de preguntar si la erotización da acceso al otro en persona. Frente al cruce de las carnes que cesa, las palabras cruzadas no pueden hacer otra cosa que ratificar el juramento. Por eso el juramento es más originario que la erotización: "La intuición de la erotización permite a nuestras carnes cruzarse, pero no reencontrarnos cada uno en persona -para ello es necesario nada menos que el significado del juramento. Por tanto, ninguno de nosotros dos goza en persona, ni de otra persona en persona. [...] entre la carne erotizada y la persona, subsiste la separación"4. Así, la erotización no alcanza a la persona en persona, sino que permanece en el puro anonimato de otro en general.

Si la carne se erotiza contra la voluntad, y no hay persona sin voluntad libre, la erotización nunca pone en juego la persona. Y si la erotización se detiene y debe volver a empezar sin afectar a nadie en persona, la repetibilidad desemboca finalmente, como tercer momento de la finitud, en la sustitución por una diversificación indefinida de otros. La sustitución significa que la erotización neutraliza la persona y por ende la naturaliza. Establece una separación entre la persona y una apariencia objetiva o natural, y con ello surge la sospecha de que no se gozaba de una persona individualizada sino de una persona en general: "Por persona entiendo aquí, en un sentido negativo, el corazón del otro, este corazón del otro, quienquiera que sea, cuya desaparición me impediría radicalmente amarlo. [...] O también, la persona tiene por función evitar que el otro se

\footnotetext{
${ }^{4}$ Ibid., p. 238.
} 
reduzca a un simple papel en la erotización, y, por tanto, exige su insustituibilidad -por la cual la persona no equivale justamente a la persona, la máscara y el papel teatral"5. Mientras que la persona exige la insustituibilidad, la erotización introduce esa separación con la apariencia que abre el espacio para el engaño y la mentira erótica.

La ausencia de persona implica que sería posible que yo no esté amando en persona en razón de que puedo recibir mi carne a pesar de mí, y de que el otro tampoco actúa en persona porque está en la misma situación de recibir su carne a pesar de él. De este modo, la erotización descalifica al juramento, es decir, compromete la posibilidad de que cada uno de los amantes diga "¡Heme aquí!".

\section{La erotización libre}

Es necesario, pues, reestablecer un nexo entre persona y erotización. Por un lado, sin la erotización, se pierde la fenomenalidad del otro porque la propia carne ya no experiencia la suya, y con ello no se puede acceder a la persona. Por el otro, este acceso a la persona se pierde si la erotización termina en una súspensión. Se debe tener en cuenta que dos fenómenos negativos muestran que la persona no desaparece totalmente: los celos contienen la exigencia fenomenológica de que el otro aparezca como una determinada persona, y el odio reivindica la persona del otro mostrando que todavía se encuentra ahí. Además, de un modo positivo, la persona se sigue mostrando, aun en la ausencia, a través de un rostro que me convoca. Por tanto, en vista de la imposibilidad tanto de pasar por alto la erotización como de renunciar a la persona, es necesario precisar el alcance de la finitud que es inherente a la erotización y conduce al ocultamiento de la persona. Esa finitud puede ser inherente no a la erotización en cuanto tal sino tan solo a la forma automática que reviste.

Marion se plantea, por tanto, el problema de si es posible una erotización de la carne que no sea automática sino libre. Una erotización libre es problemática en su efectividad porque la carne recibe lo que no depende de ella, es decir, lo que no puede realizar por sí misma. Si bien esta dependencia tornaría absurda la independencia erótica, una erotización

${ }^{5}$ Ibid., p. 240. 
voluntaria tiene sentido si se piensa en la posibilidad de tocar la otra carne y dejar tocar la mía sin contacto físico. Esta hipótesis no es absurda porque la carne no puede explicarse por algún punto en común con el cuerpo físico. Por la reducción erótica, me encuentro fuera del mundo, y, por tanto, la contigüidad espacial de las cosas es totalmente ajena a las carnes. Una carne no se adhiere a la otra como si faltara espacio físico entre ellas ni se yuxtapone a la otra como un cuerpo físico a otro. La erotización implica la experiencia de recibir la propia carne de otra carne de modo que entrar en contacto no significa en este caso otra cosa que hacer el amor en el sentido de darle su carne al otro y recibirla de él. No basta ni es necesario entrar en contacto físico con el otro para hacer el amor.

La erotización libre acontece por medio de la palabra. Se trata de una palabra muy especial porque no habla de ningún objeto o ente, sino que tiende a tocar o afectar al otro de modo que sienta la no-resistencia de mi carne. Con mi palabra, el otro puede experienciar que mi carne se le ofrece y que no va a resistirle. $\mathrm{Y}$ al escuchar puede experienciar la expansión de su propia carne de modo que goza de sí mismo por mí: "Mi palabra, que le habla del entre-nosotros, es la única que sabe tocarlo en el corazón y darle su carne a fondo -todavía sin ningún contacto espacial, que solo se volverá eventualmente lícito luego y por esta misma palabra. La palabra le da primero su carne al otro, a la distancia"6. Al hacer el amor por la sola palabra, hago el amor en persona porque depende de mí hablar o dejar de hablar. No dependo del otro porque este comienzo cae dentro de mi libertad. De modo que a mi palabra le corresponde hacer entrar al otro dentro de la reducción erótica. Marion resume de este modo las consecuencias de su análisis en relación con la amplitud de la erotización libre: "Evidentemente, puesto que se cumple sin ceder al automatismo (ni sufrir la suspensión), no se limita al ejercicio sexual del cruce de carnes. Ante ella se abre, pues, una inmensa cantera: permite dar ( $y$ recibir) una carne erotizada allí donde la sexualidad no alcanza. De padre a hijo, de amigo a amigo, de hombre a Dios. En ella se reconoce sin duda también la castidad, la virtud erótica por excelencia"?.

${ }^{6}$ Ibid., p. 281.
${ }^{7}$ Ibid., p. 283. 


\section{La fidelidad}

El fenómeno erótico requiere la prolongación del juramento, es decir, una fidelidad que remite a la eternidad. No puedo amar si limito la intención y su significado, es decir, el "iHeme aquí?" a un tiempo finito. Amar por un tiempo determinado, y aun con un mutuo acuerdo, significa no amar en absoluto. Si se convierte en una mentira, la intención de fidelidad afirmada en la expresión "iTe amo!" puede engañar al otro pero no a mí que la profeso. No es posible decir " $\mathrm{i} T$ e amo!" y excluir la obligación de fidelidad porque se trata de una contradicción performativa. Soy infiel a mí mismo porque no quiero permanecer fiel al otro. Mientras que la resolución anticipatoria según el ser está referida a la posibilidad de ser y a la posibilidad de la imposibilidad, la resolución anticipatoria en la reducción erótica abre una posibilidad que no está limitada por el ser o por la muerte, y se define como la imposibilidad de la imposibilidad. Puesto que no pertenece al horizonte del ser, y se encuentra siempre ya en la eternidad, el fenómeno erótico no desaparece con la muerte: "Así, el amante [...] alcanza una anticipación efectiva, libre y verdaderamente decidida - no anticipa más en la posibilidad de la imposibilidad (del futuro), sino en la imposibilidad de su imposibilidad. El amante, desde el comienzo de su avance, anticipa sobre la eternidad. No la desea, la presupone"8.

La resolución anticipadora me permite acceder a mi ipseidad. Fuera de ella, mi ipseidad es dudosa o inaccesible porque se la podría sustituir por otra. Según Marion, en la reducción trascendental, soy en tanto pienso según las reglas universales del pensamiento de modo que lo que pienso puede ser pensado por cualquiera. En la reducción ontológica, ser un ente al que en su ser le va tanto su ser como el de los otros entes no confiere una ipseidad última. Aun cuando dependa de mí, el ser es de todos los entes y, por tanto, se trata de un ser de entes distintos de mí. En cambio, en la reducción erótica, conozco lo mío como lo más íntimo de mí mismo. No me es posible librarme de mis avances, juramentos, etc., en razón de mi compromiso con ellos, aunque, paradójicamente, la historia erótica de mi ipseidad queda asignada a los otros: “QQué soy? A esta pregunta no tienen nada que responder ni el ser ni el ente en mí. Puesto que soy en tanto amo y

${ }^{8}$ Ibid., p. 299. 
se me ama, solo otros podrían responder. Me recibiré al final del otro, así como he nacido de él"'?

\section{El adiós}

El fenómeno erótico no solo está amenazado por la suspensión sino por el hecho de que ningún tercero, como el hijo, se mantiene el tiempo suficiente para atestiguarlo de una vez por todas. Por tanto, es necesario amar de manera tal que el próximo instante se convierta en una última instancia, es decir, en el cumplimiento mismo del destino de amante. Se ha de amar como si el próximo acto de amor fuera la última posibilidad de amar. Puesto que se sustrae a la disipación, ese instante escapa a la repetición, se torna escatológico, y se erige en el tercero que ha de atestiguar para siempre la relación erótica porque no será seguido por ningún otro instante. Con otras palabras, se ama en el último instante como si esa ocasión se tornara definitivamente la última de modo que el amante hace el amor bajo el aspecto de la eternidad: "Este vuelo inicial hacia lo definitivo se llama el adiós. Los amantes cumplen su juramento en el adiós -en el pasaje a Dios que ellos convocan como su último testigo, su primer testigo, el que no parte y no miente nunca. [...]- la próxima vez en Dios (à Dieu). Pensar en Dios puede hacerse eróticamente en este 'adiós"'10.

La resolución anticipatoria desemboca en la anticipación escatológica según la cual debo amar en el instante de un modo perfecto y para siempre. Con ella se anula la repetición y los amantes quedan instalados de entrada en el fin. Una vez amante lo soy para siempre ya que el otro atestiguará que fui su amante. Recibo del otro la dignidad de amante así como el otro la recibe de mí. Si bien no sé con seguridad que el otro me ama cuando se convierte en mi amante, sé absolutamente que me convierto en el suyo. Si bien no me convierto en amado al margen de la duda, me convierto en amante y no puedo dejar de comprobarme como tal. Así, me convalido desde el punto de vista de la reducción erótica: ' $Y$ puesto que solamente el hecho de que se me ama puede dar esta seguridad, me descubro amable en tanto amante provocado. Amado porque soy amable, amable porque soy amante"11.

\footnotetext{
${ }^{9}$ Ibid., p. 302.

${ }^{10}$ Ibid., p. 326.

${ }^{11}$ Ibid., p. 327 s.
} 
Me encuentro digno de ser amado en la medida en que el otro me asegura que cumplo la función de amante. Soy amable en tanto amante, y por eso recibo la seguridad de otra-parte. $Y$ puesto que creo que soy amable para el otro, soy también amable para mí mismo. Me amo a mí mismo gracias al otro que se revela más amante que yo. Debo al otro amante, y a su anterioridad respecto de mí, el terminar con el odio a mí mismo. Así, el otro tiene precedencia en la condición de amante.

\section{Tercera formulación de la reducción erótica}

El otro asume el rol de amante como el primero de modo que el avance se invierte y pasa de mí a él. El centro de gravedad de la reducción erótica se desplaza, y me encuentro descentrado con respecto a ella. Por tanto, la reducción no resulta de mi avance sino que, aunque yo ignorara esto, lo ha antecedido. Hay, pues, un avance de la reducción erótica sobre mi avance, y esto exige una tercera formulación: "Tú, tú me has amado el primero" 12 . No puedo dispensarme de mi propio avance porque de este modo se perpetuaría la aporía del amor a sí mismo que conduce al odio de todos contra todos. Sin embargo, descubro que este avance no me pertenece sino que me aguardaba porque el otro ha sido amante antes que yo lo he sido. Lo que yo buscaba me había encontrado ya: "Cuando avanzaba perdido en mi propio avance, avance ciego, sin saber a quién amar ni cómo, sin duda otros amantes, más antiguos que yo, me seguían con la mirada, velaban por mis pasos, y ya me amaban sin saberlo yo, a pesar de mí. Para que entre en la reducción erótica, era necesario que otro amante me hubiera precedido, $y$, desde allí me interpelara en silencio"13.

Marion observa que nunca hubiera podido preguntarme " ¿se me ama de otra-parte?" si otro no me hubiese amado en primer lugar. La lógica del amor, desencadenada por el avance, conduce a comprender que el otro me amaba mucho antes de mi avance. Nadie puede pretender que nadie lo ama ni lo ha amado. Esto se confirma con varios argumentos. En primer lugar, para quejarme de que los otros no me aman, es necesario que yo viva y para ello es necesario que otros se hayan amado uno al otro. En segundo lugar, pretender no ser amado requiere un interlocutor y entonces de presentan

\footnotetext{
12 Ibid., p. 331.

${ }^{13}$ Ibid., p. 331.
} 
dos casos. O bien el otro me escucha, y, por tanto, me ama en sentido mínimo. $\mathrm{O}$ bien nadie escucha, pero siempre queda abierta la posibilidad de que me vaya a amar un amante que todavía tiene que aparecer.

Los pasajes finales analizan la cuestión de Dios, que se nombra con el nombre mismo del amor, como amante en el horizonte de la reducción erótica: "Porque, de hecho, Dios no se revela solamente por amor y como amor, también se revela por los medios, las figuras, los momentos, los actos y los estadios del amor, del único amor y del solo amor, el que nosotros practicamos. Él hace el amante como nosotros - pasando por la vanidad (los ídolos), el pedido de que se lo ame y el avance de amar el primero, el juramento y el rostro (el ícono), la carne y el gozo de la comunión, el dolor de nuestra suspensión y la reivindicación celosa, el nacimiento del tercero en tránsito y el anuncio del tercero escatológico, que terminan identificándose con el Hijo encarnado, hasta la promulgación unilateral por él, a nosotros, de nuestra fidelidad"14. Por eso Marion se pregunta si acaso no aprendemos esta lógica de Dios. Sin embargo, hay una infinita diferencia en la condición del amor. No solo había un otro que me amaba antes de que yo lo amara, sino que ese amante era Dios: "Dios nos precede y nos trasciende, pero en primer lugar y sobre todo en esto: nos ama infinitamente mejor que nosotros amamos y lo amamos. Dios nos sobrepasa en calidad de mejor amante"15.

\section{Epílogo}

La fenomenología ha asignado desde sus comienzos un lugar central al amor: E. Husserl afirma que el amor es un problema fundamental de la fenomenología; M. Scheler observa, en "Ordo Amoris", que el hombre es un ens amans antes que un ens cogitans o un ens volens, y J. Ortega y Gasset inicia El Espectador evocando las revelaciones que nos reserva su estudio. Con estos antecedentes, limitados al terreno de la fenomenología, se empaña la afirmación inicial acerca de que la filosofía pasa por alto actualmente el tema del amor. La aportación central de Marion no reside en la reinstalación del tema sino en la delimitación y descripción de lo que, en los párrafos finales del la obra, denomina "los medios, las figuras, los momentos, los

\footnotetext{
${ }^{14}$ Ibid., p. 341.

${ }^{15}$ Ibid., p. 342.
} 
actos y los estadios del amor". Al respecto, no es aventurado especificar que (i) los medios abarcan la caricia como contacto no mundano o los diversos usos del lenguaje para dar lugar a la erotización; (ii) las figuras están representadas por el significado, el juramento, la carne, el gozo, la fidelidad y el adiós; (iii) los actos comprenden los modos de efectuación de esos medios y figuras; (iv) los estadios corresponden a las diversas formulaciones de la reducción erótica: la inicial, la radicalizada y la final; y (v) los momentos se distinguen dentro de estos estadios con la aparición de las diversas figuras. Corresponde subrayar, en el tratamiento de las dos figuras finales, una oportuna y feliz aplicación de los temas de la resolución anticipadora, el instante y el destino, derivados de M. Heidegger, a la lógica del amor.

A diferencia del tratamiento fenomenológico previo, que destaca los aspectos positivos, Marion exhibe una tendencia al rodeo por el lado negativo. Así, pasa por el amor a sí mismo para llegar al odio de todos contra todos antes de la segunda formulación de la reducción, y por la persona naturalizada para acceder a figuras de la simulación antes de la tercera formulación. Ante este procedimiento y la afirmación final de que nadie puede pretender que no ha sido amado, cabe preguntar si no es más adecuado desarrollar la lógica del amor con este fenómeno primario de donación como punto de partida. Asimismo, desde enfoques más tradicionales de la fenomenología aparecen cuestionables la noción de un fenómeno cruzado en atención al análisis de las intencionalidades en juego, la univocidad del amor en vista de la pluralidad de sus figuras, la oposición entre el cuerpo y la carne en consideración de una estratificación de lo real que mantenga los niveles previos, y la falta de explicitación del "horizonte de la erotización" que lleva a descuidar otras dimensiones relativas al mantenimiento de la persona en la suspensión del cruce de carnes.

En lo que atañe al tema de la donación, la contribución de Marion es significativa por el marcado carácter experiencial que asigna a la donación como fenómeno de doble entrada. Ofrece así una perspectiva que se asocia con, y a la vez se distingue de, otros puntos de vista en la fenomenología. Frente a P. Ricoeur, quien acentúa la mediación simbólica que el don efectúa respecto del reconocimiento mutuo, Marion insiste en el modo en que esa mutualidad se fenomenaliza en nuestra experiencia inmediata. A diferencia de B. Waldenfels, con su énfasis en el dar como respuesta a la interpelación de lo extraño y en la diferencia responsiva en virtud de la excedencia del llamado, nuestro autor equilibra esa diferencia a través del 
entrecruzamiento de dos llamados. En relación con E. Lévinas, quien presenta el dar como significancia de la responsabilidad por el otro, este análisis del fenómeno erótico se aleja -a la vez que se apropia del tema del rostro- de la responsabilidad ética universal en tanto sostiene que el amor se desenvuelve en una relación de individuo a individuo. De cara a M. Henry, con su acento en la donación de la Vida, Marion toma las nociones de carne y sentir y elabora el modo de fenomenalización de la carne del otro, pero no ofrece un análisis de la reversibilidad o irreversibilidad de las relaciones entre diferentes modalidades, divinas o humanas, de esa autoafección.

Por último, en lo que concierne a la cuestión de la subjetividad, Marion subraya -en contraste con la exhaltación hiperbólica- que recibo del otro, como una donación, mi ipseidad porque recibo mi significado en su juramento, mi carne en la erotización de su carne, y mi fidelidad cuando hace constar mi amor verdadero. Además, pone de relieve -en oposición a la destitución reductiva- que soy irreductible en tanto me individualizo y me torno radicalmente insustituible en el experienciar (se) experienciar (se) que me corresponde solo a mí.

\section{Resumen}

Este artículo procura establecer lo rasgos distintivos de los tres estadios de la reducción erótica formulada por J.-L. Marion en contraste con la reducción epistémica al cogito y la reducción ontológica al ser. En primer lugar, en la búsqueda de una seguridad que involucra al otro, la autocerteza del cogito es reemplazada por la pregunta: “ ¿se me ama de otra-parte?”. En segundo lugar, como esta pregunta no puede ser respondida recurriendo al amor a sí mismo, se impone una formulación radicalizada: “¿puedo amar, yo el primero?”. Este nuevo estadio da lugar al análisis de fenómenos en que cada participante da al otro lo que no tiene de suyo y al mismo tiempo tiene a su disposición solo para ofrecerlo al otro, esto es, el significado, la carne, el juramento, el gozo, la fidelidad, y el adiós=a Dios. En tercer lugar, puesto que esta lógica del amor muestra la prioridad del otro, se presenta la formulación final de la reducción erótica: “iTú, tú has amado el primero!”. Marion sostiene que, a través de esta precedencia, se revela el amor de Dios. Una apreciación final de las contribuciones de Marion esboza superposiciones y contrastes con otras posiciones fenomenológicas.

Palabras clave: “fenómeno erótico", “came”, “juramento”, “gozo”, “adiós=a Dios” 


\begin{abstract}
This article sets out to establish the distinctive traits of the three stages of the erotic reduction advanced by J.-L. Marion in contrast to the epistemic reduction to the cogito and the ontological reduction to Being. First, in the search for an assurance involving the other, the self-certainty of the cogito is replaced by the question " $\mathrm{iAm}$ I loved from elsewhere?". Second, as the question cannot be answered by the way of self-love, a radicalized formulation must be given: "¿Can I love as the first one?" This new stage brings forth the analysis of phenomena in which each partner gives the other what he does not have on his own and at the same time has at his disposal only to offer it to the other, i.e., meaning, flesh, pledge, joy, faithfulness, and goodby $=$ God be with you. Third, as this logic of love discloses the priority of the other, a final formulation of the reduction is afforded: " $j$ You, you have loved as the first one!" Marion contends that, through this precedence, God's love is revealed. A final assessment of Marion's contributions outlines overlappings and contrasts with other phenomenological positions.
\end{abstract}

Key words: "erotic phenomenon", “flesh", "pledge", “enjoyment", "good-by=God be with you" 